\title{
Tracking itinerant patients and surgical sponges
}

$\mathrm{H}$ ospitals in the United States have increased their spending by more than $200 \%$ since 2005 on electronic tracking systems that can locate wandering patients and misplaced equipment, detect errant blood transfusions and prevent medication errors, as well as sound alarms when surgical sponges are left in wrong places, like inside patients.

According to California-based Spyglass Consulting Group, which tracks information technologies, $76 \%$ of US hospitals with more than 300 beds have invested in radio frequency identification.

"Hospital investments in RFID [radio frequency identification systems] are taking off, especially among the larger organizations, as they already have a wireless backbone in place," says Gregg Malkary, managing director of Spyglass. "They are trying to leverage the existing investments they have made, and they are actually solving very specific business problems."

International market research firm IDTechEX substantiates this trend, noting that the health care market for radio frequency identification systems in the US, now US\$120.9 million, will soar to US\$2.03 billion within a decade.

Once used primarily to prevent infant misidentification and abduction in emergency wards, the systems are now on the wish lists of almost all hospital departments, including waiting rooms. Unlike their bar code precursors, which require line-of-sight scanning and individual handling, radio frequency identification readers can identify hundreds of tagged objects at a time, even if piled in unruly stacks, covered by surgical sheets or hidden in crumpled bedclothes. Their use has even been recommended to store medical information under the skin of patients (CMAJ 2007; 177[4]:331-2).

Radio frequency identification systems consist of 2 main components: tag and reader. Tags are basically dimesized microchips with antennas. Most are passive, like bar codes, and must be read by a reader or sensor that is either hand-held or mounted in a doorway or on a ceiling. Active tags have batteries and can emit radio signals to readers. A bar code wristband on an Alzheimer patient may be able to identify that person but, unlike a radio frequency identification band, it can't close facility doors if that person gets too close to them.

Developers claim the devices are the gold standard of tracking technology, but bar code promoters claim the radio frequency systems are too expensive to be practical. Many experts in the field who work with both technologies say using them complementarily makes the most sense: bar codes when it will suffice, radio frequency identification otherwise.

Costs are difficult to quote as they depend on what is contained in the tags, how they are encased, their transmission capabilities and other factors. But it is possible to get stripped-down tags for a dollar apiece, sometimes even a nickel.
Still, bar-coding costs but a fraction of that, less than a penny each if bought in bulk. Radio frequency identification readers are also more expensive, costing thousands instead of the hundreds required to buy a bar code reader.

Despite the added expense, some hospitals have embraced the technology. The gastroenterology and colorectal surgery outpatient endoscopy unit at the Mayo Clinic has reported that since it began labelling specimen bottles with radio frequency identification tags, errors due to mislabelling dropped precipitously: from 765 errors on 8231 bottles sent to pathology in the first quarter of 2007 , to only 47 on 8539 bottles in the first quarter of 2008 .

Dr. Schuyler Sanderson, a Mayo pathologist, says that "the elimination of the paper requisition form, which [required] anywhere from 1 to $5 \mathrm{~min}$ utes, has now been given back to our nursing staff so they can directly return to patient care. ... Before we started this project we were looking at between

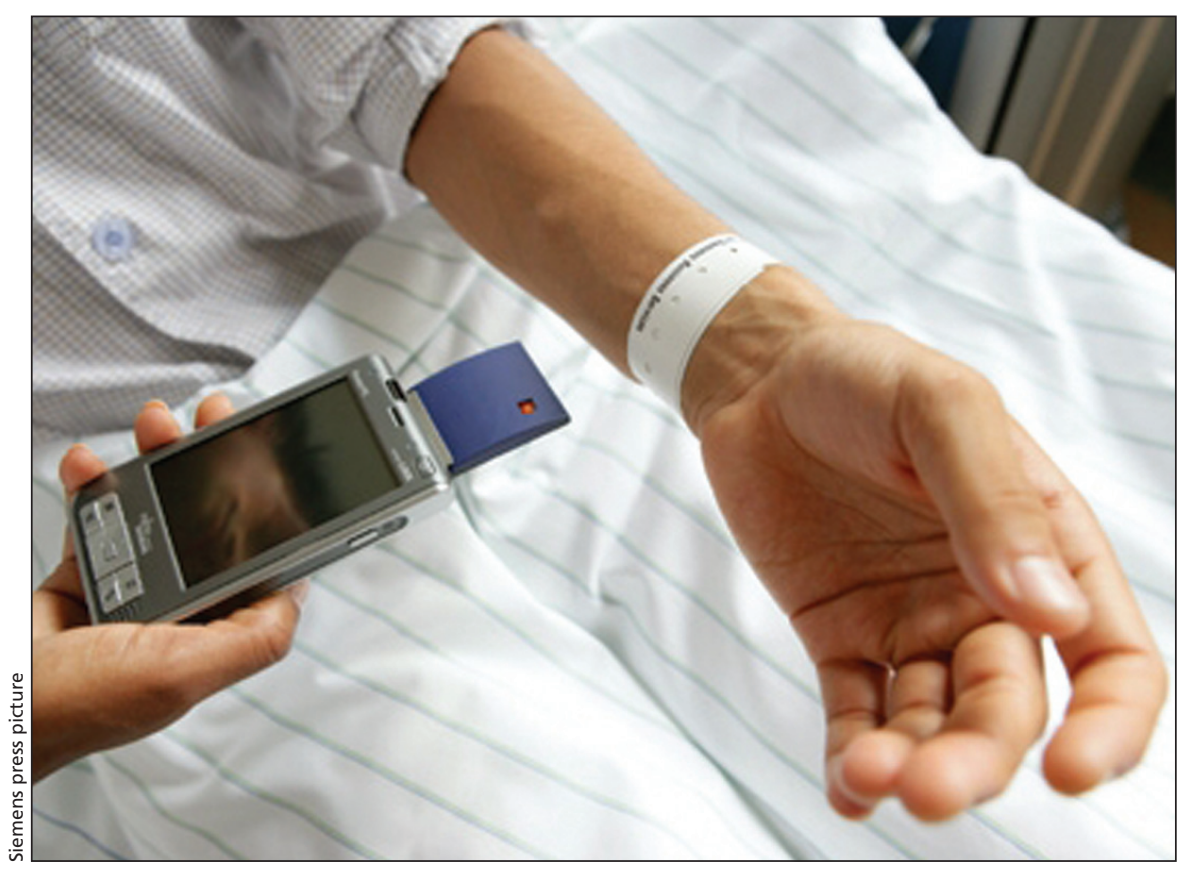

Many hospitals in the United States are discovering that radio frequency identification systems are superior to older technologies for keeping track of everything from patients to sponges. 
US\$120 000 and US\$125000 spent annually in personnel costs tracking down errors. Now, virtually zero."

The Albert Einstein Healthcare Network in Philadelphia, Pennsylvania, deployed a passive radio frequency identification tracking system in 2003 and has since increased emergency department volume by $24 \%$ thanks to better traffic flow. The number of patients who leave before getting treated has dwindled from $5 \%$ to $1 \%$.

In 2008, ClearCount Medical Solutions, a Pennsylvania-based company, introduced its SmartSponge system, which includes a tray with a radio frequency identification reader that counts the number of sponges set out at the start of operations, counts those discarded into its SmartBucket during and after surgery, and confirms if the numbers match. The tagged-sponge system costs about US\$19 000, modest compared to surgical do-overs or litigation. In 2007, for example, a Georgia court awarded US\$10 million to a woman who had a surgical sponge left inside her after colon surgery.

Hospitals in the United Kingdom and Europe are also aggressively pursuing the technology. Providers such as Oracle and Intel estimate the market for the technology in European health care to be about US\$420 million. The National Health Service is investing especially heavily in safeguarding blood transfusions through tagging.

But various consumer groups and government agencies have expressed privacy concerns about the tracking of hospital patients and staffs and the potential for highly sensitive patient information being hacked or used without patient knowledge. The National
Institute of Standards and Technology, in its Guidelines for Securing RFID Security, has also raised red flags, not only about privacy but about the possibility that the radio frequencies used by the systems could interfere with wireless patient sensors, medical telemetry devices and other medical technologies. One recent study indicated that, in test settings, radio waves from radio frequency identification devices sometimes switched off ventilators, stopped syringe pumps and caused external pacemakers and dialysis machines to malfunction. But researchers noted that most malfunctions occurred only when active tags were placed within a foot of equipment (JAMA 2008;299[24]:2884-90). — Milan Korcok, Fort Lauderdale, Florida

DOI:10.1503/cmaj.090034 